\title{
Recent Results from Photoproduction of Mesons in A2
}

\author{
Natalie Walford ${ }^{1, \star}$ \\ for the A2 Collaboration \\ ${ }^{1}$ University of Basel (Basel, Switzerland)
}

\begin{abstract}
Recent experiments using the Crystal Ball/TAPS setup at the MAMI accelerator in Mainz, Germany continue to study the properties and the excitation spectrum of the nucleon with meson photoproduction. Electromagnetic excitations of the proton and neutron are essential for understanding their isospin decomposition. The electromagnetic coupling of photons to protons is different than that of neutrons in certain states. Hence, a complete partial wave analysis (PWA) can assist in yielding more information about any reaction, but gains from polarization observables constraining the fits. Polarization observables play a crucial role as they are essential in disentangling the contributing resonant and non-resonant amplitudes, whereas cross-section data alone is not sufficient for separating resonances. Preliminary results of polarization observables $(E, T$, and $F)$ of $\eta$ and double $\pi$ production off a polarized neutron (dButanol) target are shown with comparison to predictions of recent multipole analyses. These results will allow for developing the world database.
\end{abstract}

\section{Introduction}

A complete picture of the spectrum of $N^{*}$ resonances is essential to understanding the excitation spectrum of the nucleon. For many years now, the study of the nucleon and its excitation spectrum has been studied. Understanding the dynamics and relevant degrees of freedom within hadrons in the non-pertubative regime of quantum chromodynamics (QCD) is crucial, as QCD describes the strong force acting between quarks, which currently can only be solved at very low and high energies where perturbative methods can be used.

Approximation models describe the behaviour of quarks in nucleons at medium energies. Meson photoproduction can specifically allow for unique possibilities to investigate these nucleons and their excited states (or resonances). Unfortunately, many states are overlapping and cannot be easily differentiated from each other and many have been predicted, but not yet detected. [1] The states that have not yet been observed could be missing because they have not been seen experimentally until now or do not exist at all. Most earlier experiments were preformed with pion beams and some resonances might couple less strongly to pions and couple more strongly to rare channels and have circumvented detection. Most results still arise from experiments on the proton, which does not allow for as much information regarding the isospin structure of the electromagnetic transitions. Therefore, advances in

^e-mail: natalie.walford@unibas.ch 
experiments on the neutron can provide an integral piece to the understanding of the nucleon spectrum. Unfortunately, it is not possible to perform measurements on the free nuetron, but a deuterated butanol target has allowed for the possibility to study spin effects with quasi-free neutrons.

For years, cross section data has been used to learn about the nucleon spectrum. However, cross section data alone is not enough to distinguish the broad overlapping resonances. Polarization observables can provide understanding of these overlapping resonances by discovering more information about the complex helicity amplitudes, which describe the interaction between photon beams and nucleons. These amplitudes are fully determined when a complete set of measurements is performed and give rise to the cross section, complemented by polarization observables including beam, target, and recoil asymmetries. The following observables will be discussed: $E$ (longitudinally polarized target and circularly polarized photon beam), $F$ (transversely polarized target and circularly polarized photon beam), and $T$ (transversely polarized target and an unpolarized photon beam).

The relationship between the cross section and polarization observable $E$ can be written in terms of the helicity of beam and target as follows:

$$
E=\frac{\sigma_{1 / 2}-\sigma_{3 / 2}}{\sigma_{1 / 2}+\sigma_{3 / 2}}=\frac{\sigma_{1 / 2}-\sigma_{3 / 2}}{2 * \sigma_{0}}
$$

where $\sigma_{1 / 2}$ is the cross section of events with anti-parallel beam and target polarizations with total spin $\frac{1}{2}$ and $\sigma_{3 / 2}$ is the cross section of events with parallel beam with total spin of $\frac{3}{2}$. Due to the page limit, the $T$ and $F$ observable, which have a similar equation, will not be shown, but were discussed during the workshop.

\section{Experimental Setup}

Data described herein is from experiments performed at the MAMI-C accelerator in Mainz, Germany [2]. A longitudinally polarized electron beam of energy $1557 \mathrm{MeV}$ and polarization degree of $80 \%$ is delivered into the A2 experimental Hall. Circularly polarized photons are produced from a radiator and energy tagged using the Glasgow-Mainz photon tagger [3] with energies between 470 and 1450 $\mathrm{MeV}$. The targets consists of a deuterated butanol material (dButanol or $D_{9} C_{4} O D$ ) cooled to a low temperature with the deuterons either transversally or longitudinally polarized up to $80 \%$. The target is surrounded by a cylindrical particle identification detector (PID) made up of 24 plastic scintillator strips that each cover $15^{\circ}$ in the azimuthal angle. The PID is then surrounded by a multi-wire poroportional chamber (MWPC) and the MWPC is surrounded by the spherical Crystal Ball (CB) calorimeter [4]. The $\mathrm{CB}$ consists of $672 \mathrm{NaI}(\mathrm{Tl})$ crystals and covers $20^{\circ}$ to $160^{\circ}$ in the polar angle. In the forward direction, a hexagonal two arm photon spectrometer (TAPS) built from $72 \mathrm{PbWO}_{4}$ (two innermost rings) and $366 \mathrm{BaF}_{2}$ crystals (remaining rings) is present. A veto wall is present in front of TAPS is used for particle identification. The combination of the CB and TAPS provides an almost $4 \pi$ acceptance in the center of mass fram with a high angular and energy resolution.

\section{Analysis and Results}

Using information from the detectors, events are collected and then selected based on the number of charged or neutral hits. Neutral mesons are identified via a $\chi^{2}$ test, which tries to the find the best combination of photon clusters for the meson invariant mass. To eliminate accidental conincident tagger photons, coincidence time cuts are applied and random background subtraction was performed. To separate the background from the signal, kinematic cuts were applied for each $W$ and $\cos \theta \operatorname{bin}$. Relevant events come from the polarized deuterons with cuts applied, but events from carbon and 
oxygen are subtracted using a separate beam time on a carbon $\left({ }^{12} C\right)$ target with similar experimental conditions. The subtraction of reactions on unpolarized carbon and oxygen nuclei inside the dButanol target was performed. By comparing the missing mass spectra of the dButanol, liquid deuterium, and carbon beam times, allows for further studies of the contributions of reactions on unpolarized nuclei.

Different versions were used to extract the final $E$ asymmetry. Version 1 refers to where the asymmetry was normalized with twice the unpolarized cross section $\sigma_{0}$, which was measured with a liquid deuterium target, and does not need to utilize carbon background subtraction since the unpolarized background cancels in the difference of the two helicity states $\left(\sigma_{\Delta}=\sigma_{1 / 2}-\sigma_{3 / 2}\right)$. Version 2 refers to the normalization of the numerator using the sum of the two spin configurations $\left(\sigma_{\Sigma}=\sigma_{1 / 2}+\sigma_{3 / 2}\right)$. Here, the background from unpolarized carbon and oxygen nuclei inside the target have to be subtracted, allowing for events only on polarized protons and neutrons. Version 3 refers to using the sum and difference of the two helicity states to calculate $E$ and the carbon was used to subtract the unpolarized carbon and oxygen nuclei. Finally, version 4 refers to using a direct calculation of $\sigma_{1 / 2}$ and $\sigma_{3 / 2}$. An overview of the different versions are summarized in Table 1 .

Table 1. Overview of the versions used to extract the $E$ asymmetry.

\begin{tabular}{|lccc|}
\hline Version & $E$ & $\sigma_{1 / 2}$ & $\sigma_{3 / 2}$ \\
\hline 1 & $\frac{\sigma_{\Delta}}{2 * \sigma_{0}}$ & $\sigma_{0}(1+E)$ & $\sigma_{0}(1-E)$ \\
2 & $\frac{\sigma_{\Delta}}{\sigma_{\Sigma}}$ & $\sigma_{0}(1+E)$ & $\sigma_{0}(1-E)$ \\
3 & & $\frac{\sigma_{\Sigma}+\sigma_{\Delta}}{2}$ & $\frac{\sigma_{\Sigma}-\sigma_{\Delta}}{2}$ \\
4 & $\frac{\sigma_{1 / 2}-\sigma_{3 / 2}}{\sigma_{1 / 2}+\sigma_{3 / 2}}$ & $\sigma_{1 / 2}$ & $\sigma_{3 / 2}$ \\
\hline
\end{tabular}

Fig. 1 shows the results for $\gamma p \rightarrow p \eta$ and $\gamma n \rightarrow n \eta$ for $E, \sigma_{1 / 2}$, and $\sigma_{3 / 2}$. For the $E$ asymmetry, it is expected to be at unity close to threshold due to the dominance of the $N(1535) 1 / 2^{-}$. This is seen in the results off the neutron and the two extraction versions are in good agreement. A deviation occurs in the proton data of $E$, but is related to the detection efficiency for protons. The results of $E$ are in good agreement with the BnGa model [6], but differ from MAID [5] due to the unrealistic contribution of $N(1675) 5 / 2^{-}$in the model. For the $\sigma_{1 / 2}$ proton results, they are in reasonable agreement with model predictions. An enhancement is seen around $W \approx 1720 \mathrm{MeV}$ for the $\sigma_{3 / 2}$ proton component. For the $\sigma_{1 / 2}$ neutron results, a narrow structure is seen around $W \approx 1670 \mathrm{MeV}$, which is related to the $J=1 / 2$ partial wave and appears to be a contribution from a narrow $P_{11}$ resonance.

Results for $\gamma p \rightarrow p \pi^{0}$ and $\gamma n \rightarrow n \pi^{0}$ for $E, \sigma_{1 / 2}$, and $\sigma_{3 / 2}$ are shown in Fig. 2. Here, it can be seen that the individual contributions to $\sigma_{1 / 2}$ and $\sigma_{3 / 2}$ in the second and third resonance region are under- or overestimated by the models. It is worth noting that the MAID [8] model achieves the poorest agreement. SAID [7] and BnGa [9] are, apart from $E$ on the neutron (where SAID achieves the better agreement), are very similar to each other and agree with the shape of the data quite well. Due to page limitations, the double $\pi^{0}$ results have been omitted, but were discussed in the workshop.

\section{Conclusion}

In conclusion, these measurements of coincident neutrons in neutral final states are crucial for the analysis of $N^{*}$ properties. Due to the lack of neutron data in the world database, these results will provide insight into constraining the current PWA models and help to discover all the states of the nucleon. 

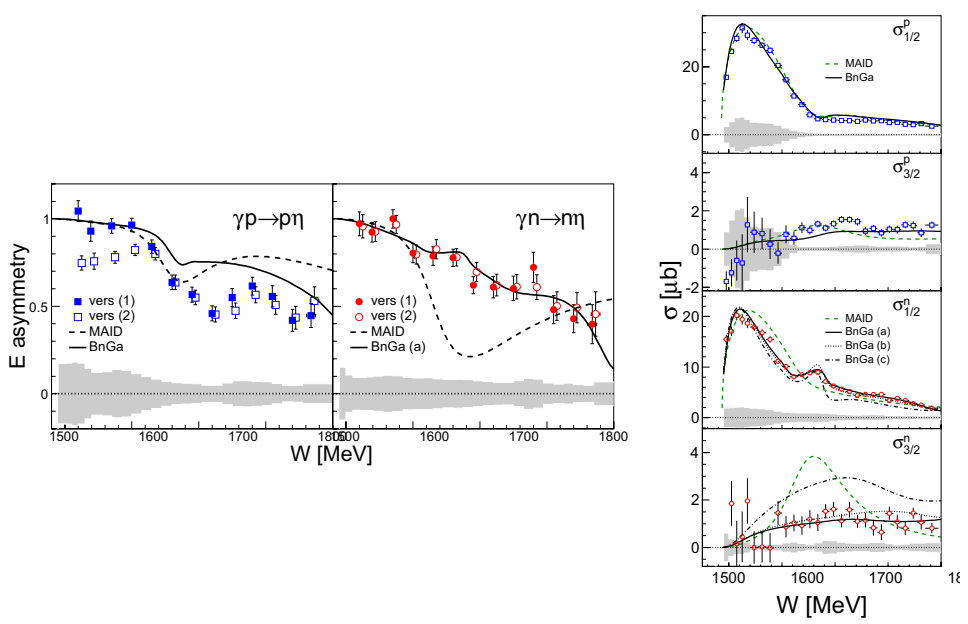

Figure 1. Double polarization observable $E$ for $\gamma p \rightarrow p \eta$ (left) and $\gamma n \rightarrow n \eta$ (right). Solid symbols: Version 1, open symbols: Version 2, gray shaded areas: systematic uncertainties. Curves: predic- tions from MAID (dashed) [5] and $\mathrm{BnGa}$ (model based on $S_{11}$ interference) [6]. Total cross sections are also shown. $\gamma p \rightarrow p \eta$ (blue squares) and $\gamma n \rightarrow n \eta$ (red circles), gray shaded areas (systematic uncertainties). Curves: model predictions from MAID (dashed green) [5], $\mathrm{BnGa}(\mathrm{a})$ (model version with interference in $S_{11}$ wave, solid black) [6], $\mathrm{BnGa}$ (b) (model with narrow $P_{11}$ resonance with positive A1/2 coupling, dotted black) [6], and $\mathrm{BnGa}$ (c) (narrow $P_{11}$ resonance with negative $A_{\frac{1}{2}}$ coupling, dash-dotted, black) [6].

\section{Acknowledgments}

All results presented in this paper have been obtained in the framework of the A2 collaboration at the Mainz MAMI accelerator. This work was supported by Swiss National Fund (SNF) and Deutsche Forschungsgemeinschaft (DFG).

\section{References}

[1] R.G. Edwards et al., Phys. Rev. D84 074508 (2011)

[2] K. H. Kaiser et al., NIM A593 159 (2008)

[3] J. C. George et al., Eur. Phys. J. A37 129 (2008)

[4] A. Starostin et al., Phys. Rev. C64 055205 (2001)

[5] W.-T. Chiang et al., Nucl. Phys. A 700, 429 (2002)

[6] A.V. Anisovich et al., Eur. Phys. J. A 51, 72 (2015)

[7] R.L. Workman et al., Phys. Rev. C86, 015202 (2012)

[8] D. Drechsel et al., Nucl. Phys. A 645, 145 (1998)

[9] E. Gutz et al., Eur. Phys. J. A50 74 (2014) 

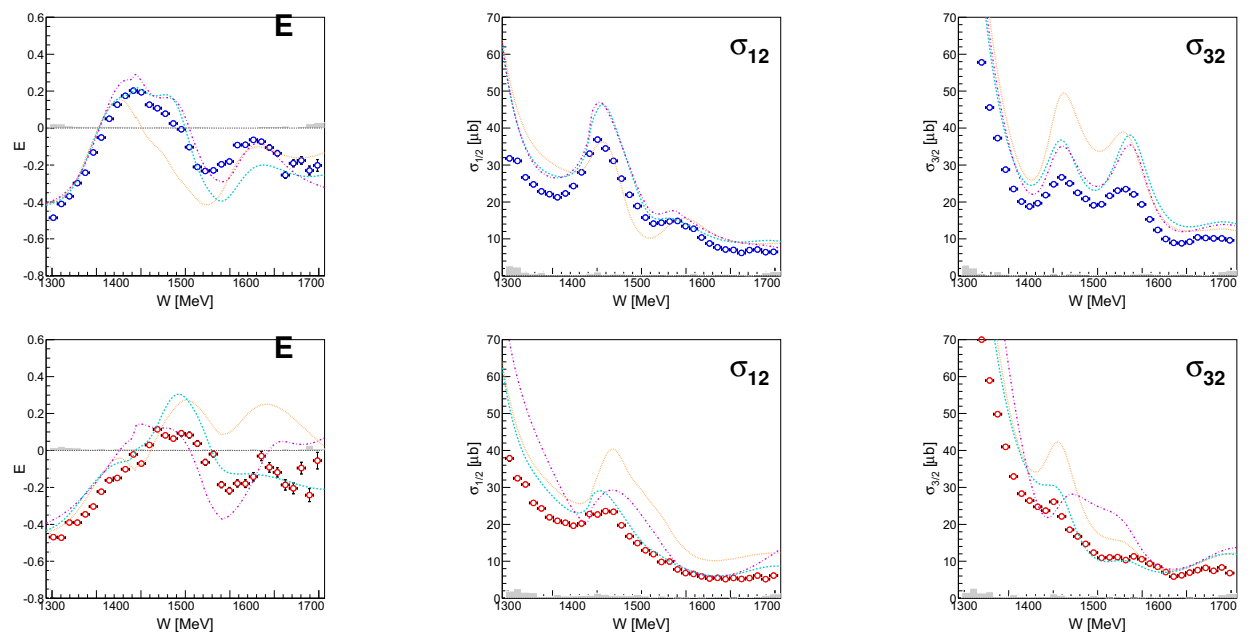

Figure 2. $E$ (first column), $\sigma_{1 / 2}$ (middle column), and $\sigma_{3 / 2}$ (last column) asymmetries for single $\pi^{0}$ for quasifree protons (upper row) and quasi-free neutrons (lower row). The cyan lines are SAID [7] nucleon models, the orange lines are MAID [8] nucleon models, and the magenta lines are Bonn-Gatchina [9] nucleon models. 